\title{
A unified approach to define fuzzy integrals
}

\author{
Luis M. de Campos, María T. Lamata and Serafín Moral \\ Departamento de Ciencias de la Computacion e I.A., Universidad de Granada, 18071 Granada, \\ Spain
}

Received February 1989

Revised May 1989

Abstract: Recently, the two most general and well-known fuzzy integrals (Sugeno's integral and Choquet's integral) were characterized. It was also shown how both integrals fitted the same formal model, and they only differed in the operators used. In this paper we try to analyze whether it is possible to improve those characterization theorems, and we also study the possibility of defining other fuzzy integrals following the same model but using other operators.

Keywords: Measure theory; fuzzy integrals; fuzzy measures.

\section{Introduction}

In a recent paper, Campos and Bolaños [6] characterized two general and well-known fuzzy integrals: Sugeno's integral [11] and Choquet's integral [7], the last one also called monotone expectation $[2,4,5,6]$.

The two characterization theorems are based on the same formal model of four properties, which only differ in the operators used: sum and product for Choquet's integral, maximum and minimum for Sugeno's integral.

Two important questions remained open: one involved the characterization theorems of these integrals in an attempt to further refine them; the second question was to investigate the possibility of defining other fuzzy integrals using different operators but following the same model. In this paper we give answers to those problems.

Section 2 is devoted to describing fuzzy integrals (defined on Sugeno's fuzzy measures), particularly Sugeno's and Choquet's integrals, and their characterizations.

In Section 3, we show how the characterization of Choquet's integral can be refined, whereas the same cannot be made for Sugeno's integral.

Finally, other fuzzy integrals fitting our formal model are obtained in Section 4.

\section{Fuzzy measures and integrals}

Let $X=\left\{x_{1}, x_{2}, \ldots, x_{n}\right\}$ be a finite referential. A fuzzy measure on $X$ in Sugeno's sense [11] is a set function

$$
g: \mathscr{P}(X) \rightarrow[0,1]
$$

0165-0114/91/\$03.50 C 1991-Elsevier Science Publishers B.V. (North-Holland) 
satisfying:

(i) $g(\emptyset)=0, g(X)=1$.

(ii) $A \subseteq B \Rightarrow g(A) \leqslant g(B)$.

Fuzzy measures are powerful tools to represent certain types of non-additive but monotone gradations, such as the ones including human subjectivity. For example, degrees of importance or relevance (e.g. relevance degrees of sets of symptoms to diagnose an illness), degrees of belief or confidence (e.g. confidence degrees about the membership to each subset of $X$ of an unknown variable taking its values on $X$ ). So, fuzzy measures are useful to represent subjective, uncertain and/or imprecise states of knowledge. We denote by $\mathcal{M}$ the class of fuzzy measures on $X$.

When a fuzzy measure is available on $X$, it is interesting to have tools capable of summarizing all the values of a function to a single point, in terms of the underlying fuzzy measure. These tools are the fuzzy integrals.

Given a class of functions $\mathscr{F} \subseteq\{h: X \rightarrow \mathbb{R}\}$ and a class of fuzzy measures $\mathscr{S} \subseteq \mathcal{M}$, a functional

$$
I: \mathscr{F} \times \mathscr{S} \rightarrow \mathbb{R}, \quad h, g \rightarrow I(h, g),
$$

is a fuzzy integral.

There are many types of fuzzy integrals although we will only consider those that, unlike others $[1,8,12,14]$, can be defined on any fuzzy measure; that is, we will only study the case $\mathscr{S}=\mathscr{M}$.

Fuzzy integrals can be useful in various senses: for example, in Decision Theory, if the available information about the states of nature is given by means of a fuzzy measure, fuzzy integration of the payoff function gives an average value that could be used to determine the best alternative [3].

In the above mentioned example of diagnosing illnesses, symptoms could be partially satisfied (e.g. the fever may be 'intense' or 'moderate', . . . ). If we assign a number to each symptom representing the degree of verification of such symptom, fuzzy integration of the function made in this way would represent relevance of this fuzzy set of symptoms.

The two fuzzy integrals that we consider more useful are:

(a) Choquet's integral $[2,7]:$ Let $\mathscr{F}_{\infty}=\left\{h: X \rightarrow \mathbb{R}_{0}^{+}\right\}$. If $h \in \mathscr{F}_{\infty}$ and $g \in \mathcal{M}$, Choquet's integral of $h$ with respect to $g$ is

$$
E_{g}(h)=\int_{0}^{+\infty} g\left(H_{\alpha}\right) \mathrm{d} \alpha,
$$

where $H_{\alpha}=\{x \in X \mid h(x) \geqslant \alpha\}$.

(b) Sugeno's integral [11]: Let $\mathscr{F}_{1}=\{h: X \rightarrow[0,1]\}$. If $h \in \mathscr{F}_{1}$ and $g \in \mathcal{M}$, Sugeno's integral of $h$ with respect to $g$ is

$$
S_{g}(h)=\max _{0 \leqslant \alpha \leqslant 1}\left\{\min \left(\alpha, g\left(H_{\alpha}\right)\right)\right\} .
$$

To characterize the previous fuzzy integrals, Campos and Bolaños [6] defined the concept of equiordered functions:

Given two real functions $h$ and $h^{\prime}$ on $X, h$ is said to be equiordered with $h^{\prime}$, 
denoted by $h \simeq h^{\prime}$, if and only if $h$ is a constant function or, for each pair $x_{i}, x_{j} \in X$ such that $h\left(x_{i}\right)<h\left(x_{j}\right)$, then $h^{\prime}\left(x_{i}\right) \leqslant h^{\prime}\left(x_{j}\right)$.

This definition attempts to reflect the idea that the values of both functions are ordered in the same way. [6]:

Starting from the equiordering relation, the following theorems were proved

Theorem 2.1. A functional $1: F_{\infty} \rightarrow \mathbb{R}_{0}^{+}$satisfies the conditions:

$\left(\mathrm{a}^{+}\right) h \simeq h^{\prime} \Rightarrow I\left(h+h^{\prime}\right)=I(h)+I\left(h^{\prime}\right)$ (ordered additivity),

(b) $h \leqslant h^{\prime} \Rightarrow I(h) \leqslant I\left(h^{\prime}\right)$,

(c) $I\left(I_{X}\right)=1$, where $I_{X}$ is the constant function equal to 1 ,

(d) $I(a h)=a I(h) \forall a \in \mathbb{R}^{+}$,

if and only if there exists only one fuzzy measure $g$ such that $I(\cdot)$ is Choquet's integral with respect to $g, E_{g}(\cdot)$.

Theorem 2.2. A functional $I: \mathscr{F}_{1} \rightarrow[0,1]$ satisfies the conditions

$\left(\mathrm{a}^{\vee}\right) h \simeq h^{\prime} \Rightarrow I\left(h \vee h^{\prime}\right)=I(h) \vee I\left(h^{\prime}\right)$ (ordered F-additivity),

(b), (c) and

$\left(\mathrm{d}^{\wedge}\right) I(a \wedge h)=a \wedge I(h) \forall a \in(0,1]$,

if and only if there exists only one fuzzy measure $g$ such that $I(\cdot)$ is Sugeno's integral with respect to $\mathrm{g}, S_{g}(\cdot)$.

In these theorems the fuzzy measure $g$ was constructed as $g(A)=I\left(I_{A}\right) \forall A \subseteq X$, where $I_{A}$ is the characteristic function of $A$.

So, both fuzzy integrals fit analogous models, and they only differ in the operators used: sum and product for Choquet's integral, maximum and minimum for Sugeno's integral.

Moreover the expressions of $S_{g}(h)$ and $E_{g}(h)$ are:

$$
\begin{aligned}
& S_{g}(h)=\bigvee_{i=1}^{n}\left(h\left(x_{i}\right) \wedge g\left(A_{i}\right)\right), \\
& E_{g}(h)=\sum_{i=1}^{n}\left(\left(h\left(x_{i}\right)-h\left(x_{i-1}\right)\right) g\left(A_{i}\right)\right), \\
& E_{g}(h)=\sum_{i=1}^{n}\left(h\left(x_{i}\right)\left(g\left(A_{i}\right)-g\left(A_{i+1}\right)\right)\right),
\end{aligned}
$$

where $h\left(x_{1}\right) \leqslant h\left(x_{2}\right) \leqslant \cdots \leqslant h\left(x_{n}\right), A_{i}=\left\{x_{i}, x_{i+1}, \ldots, x_{n}\right\}, i=1, \ldots, n, A_{n+1}=$ $\emptyset$ and $h\left(x_{0}\right)=0$.

\section{Improving the characterization theorems}

In this section we study the possibility of improving the above characterizations: is it possible to remove some of the conditions without compromising the results? 
We will start with Choquet's integral. We will see how conditions $\left(\mathrm{a}^{+}\right),(\mathrm{b})$ and (c) are indispensable. For that we will consider examples of functionals satisfying all the conditions but one $\left(\left(\mathrm{a}^{+}\right)\right.$, (b) or $\left.(\mathrm{c})\right)$, and we will prove that this one cannot be deduced. Next, we will prove that the condition $\left(d^{\circ}\right)$ is a consequence of conditions $\left(\mathrm{a}^{+}\right),(\mathrm{b})$ and $(\mathrm{c})$.

Example 3.1. Let us consider the functional $I(\cdot)$ defined as

$$
I(h)=\left(\prod_{i=1}^{n} h\left(x_{i}\right)\right)^{1 / n}
$$

It is evident that $I(\cdot)$ satisfies conditions (b), (c) and $\left(\mathrm{d}^{*}\right)$. Nevertheless $I(\cdot)$ does not satisfy $\left(\mathrm{a}^{+}\right)$: For example, if

$$
\begin{aligned}
& h\left(x_{i}\right)=0.5, i=1, \ldots, n \Rightarrow I(h)=0.5, \\
& h^{\prime}\left(x_{1}\right)=0, h^{\prime}\left(x_{j}\right)=0.5, j=2, \ldots, n \Rightarrow I\left(h^{\prime}\right)=0, \\
& h+h^{\prime}\left(x_{1}\right)=0.5, h+h^{\prime}\left(x_{i}\right)=1, i=2, \ldots, n \Rightarrow I\left(h+h^{\prime}\right)=(0.5)^{1 / n} .
\end{aligned}
$$

Obviously $h$ is equiordered with $h^{\prime}$ but

$$
I\left(h+h^{\prime}\right)=(0.5)^{1 / n} \neq 0.5=I(h)+I\left(h^{\prime}\right) .
$$

So, the condition $\left(\mathrm{a}^{+}\right)$is not a consequence of (b), (c) and (d').

Example 3.2. Let us consider the functional

$$
I(h)=h\left(x_{1}\right)+\max _{1 \leqslant i \leqslant n} h\left(x_{i}\right)-\min _{1 \leqslant i \leqslant n} h\left(x_{i}\right) .
$$

It can be easily checked that $I(\cdot)$ satisfies conditions $\left(\mathrm{a}^{+}\right),(\mathrm{c})$ and $\left(\mathrm{d}^{\bullet}\right)$. However $I(\cdot)$ does not satisfy $(\mathrm{b})$ : For example, if

$$
\begin{aligned}
& h\left(x_{1}\right)=1, h\left(x_{i}\right)=0, i=2, \ldots, n \Rightarrow I(h)=2, \\
& h^{\prime}\left(x_{j}\right)=1, j=1, \ldots, n \Rightarrow I\left(h^{\prime}\right)=1 .
\end{aligned}
$$

Then $h \leqslant h^{\prime}$ but $I(h)=2>1=I\left(h^{\prime}\right)$. Therefore the condition (b) cannot be deduced from $\left(a^{+}\right),(c)$ and $\left(d^{*}\right)$.

Example 3.3. Let $I(\cdot)$ be the functional

$$
I(h)=\sum_{i=1}^{n} \lambda_{i} h\left(x_{i}\right)
$$

where $\lambda_{i} \geqslant 0, i=1, \ldots, n$ and $\sum_{i=1}^{n} \lambda_{i}>1$.

It is obvious that $I(\cdot)$ satisfies the conditions $\left(\mathrm{a}^{+}\right),(\mathrm{b})$ an $\left(\mathrm{d}^{\cdot}\right)$ whereas it does not satisfy (c). 
So the conditions $\left(\mathrm{a}^{+}\right)$, (b) and (c) of the Theorem 2.1 are indispensable to characterize Choquet's integral. Now we prove that the condition $\left(d^{*}\right)$ is not essential:

Proposition 3.1. Every functional on $\mathscr{F}_{\infty}$ fulfilling the conditions $\left(\mathrm{a}^{+}\right),(\mathrm{b})$ and $(\mathrm{c})$ satisfies the condition $\left(\mathrm{d}^{*}\right)$ too.

Proof. We must prove that $I(r h)=r I(h) \forall r \in \mathbb{R}^{+}$. We distinguish several cases:

1. If $r$ is a natural number $(r \in \mathbb{N})$, then

$$
I(r h)=I(h+\cdots+h)=I(h)+\cdots+I(h)=r I(h),
$$

because $I(\cdot)$ fulfills $\left(\mathrm{a}^{+}\right)$and $h \simeq h$. As $I(h)=I(r(1 / r) h)=r I((1 / r) h)$, then $I((1 / r) h)=(1 / r) I(h)$.

2. If $r$ is a positive rational number $\left(r \in \mathbb{Q}^{+}\right), r=p / q$ where $p, q \in \mathbb{N}$, then because of case 1 ,

$$
I(r h)=I\left(\frac{p}{q} h\right)=p I\left(\frac{1}{q} h\right)=\frac{p}{q} I(h)=r I(h) .
$$

So, from condition $\left(\mathrm{a}^{+}\right)$we can deduce the condition $\left(\mathrm{d}^{*}\right)$ for positive rational numbers.

3. If $r \in \mathbb{R}^{+}, r$ is the limit of a non-decreasing sequence $\left\{r_{n}\right\}$ of positive rational numbers. In this case we have

$$
I(r h)=\lim _{n \rightarrow \infty} I\left(r_{n} h\right)=\lim _{n \rightarrow \infty}\left(r_{n} I(h)\right)=\left(\lim _{n \rightarrow \infty} r_{n}\right) I(h)=r I(h)
$$

Then, it suffices to prove

$$
I(r h)=\lim _{n \rightarrow \infty} I\left(r_{n} h\right) .
$$

Let $c=\max _{1 \leqslant i \leqslant n} h\left(x_{i}\right)$; as $c>0$ (except for the trivial case in which $h\left(x_{i}\right)$ is always equal to zero) and $r_{n} \uparrow r$, we can assure that

$$
\forall \varepsilon>0 \exists n_{0} \in \mathbb{N} \text { such that for } n \geqslant n_{0}, \quad\left|r-r_{n}\right|=r-r_{n}<\frac{\varepsilon}{c} .
$$

Then

$$
r h\left(x_{i}\right)-r_{n} h\left(x_{i}\right)=\left(r-r_{n}\right) h\left(x_{i}\right)<\frac{\varepsilon}{c} h\left(x_{i}\right) \leqslant \varepsilon, \quad i=1, \ldots, n .
$$

So

$$
r h\left(x_{i}\right)<r_{n} h\left(x_{i}\right)+\varepsilon, \quad i=1, \ldots, n .
$$

If we use conditions (b) and $\left(\mathrm{a}^{+}\right)$,

$$
I(r h) \leqslant I\left(r_{n} h+\varepsilon\right)=I\left(r_{n} h\right)+I(\varepsilon) .
$$

On the other hand, without loss of generality, we can suppose that $\varepsilon$ is a rational 
number (because $\mathbb{Q}^{+}$is a dense set on $\mathbb{R}^{+}$), and then, using condition (c),

$$
I(\varepsilon)=I\left(\varepsilon I_{X}\right)=\varepsilon I\left(I_{X}\right)=\varepsilon
$$

Therefore

$$
\left|I(r h)-I\left(r_{n} h\right)\right|=I(r h)-I\left(r_{n} h\right) \leqslant \varepsilon
$$

and expression (4) is proved.

So, a more refined Choquet's integral characterization theorem is:

Theorem 3.1. A functional $I: \mathscr{F}_{\infty} \rightarrow \mathbb{R}_{0}^{+}$satisfies the conditions

$\left(\mathrm{a}^{+}\right) h \simeq h^{\prime} \Rightarrow I\left(h+h^{\prime}\right)=I(h)+I\left(h^{\prime}\right)$,

(b) $h \leqslant h^{\prime} \Rightarrow I(h) \leqslant I\left(h^{\prime}\right)$,

(c) $I\left(I_{X}\right)=1$,

if and only if there exists a unique fuzzy measure $g$ such that $I(\cdot)$ is Choquet's integral with respect to $g, E_{g}(\cdot)$.

In the case of Sugeno's integral we can remove no condition, as is shown in the following examples:

Example 3.4. Let $I(\cdot)$ be the functional defined by

$$
I(h)=\min \left(\max _{1 \leqslant i \leqslant n} h\left(x_{i}\right), 2 \min _{1 \leqslant i \leqslant n} h\left(x_{i}\right)-\left(\min _{1 \leqslant i \leqslant n} h\left(x_{i}\right)\right)^{2}\right) .
$$

Some calculations suffice to prove that $I(\cdot)$ satisfies the conditions (b), (c) and $\left(d^{\wedge}\right)$. However $I(\cdot)$ does not satisfy $\left(a^{\vee}\right)$ : For example, if

$$
\begin{aligned}
& h\left(x_{i}\right)=0.6, i=1, \ldots, n \Rightarrow I(h)=0.6, \\
& h^{\prime}\left(x_{1}\right)=1, h^{\prime}\left(x_{j}\right)=0, j=2, \ldots, n \Rightarrow I(h)=0, \\
& h \vee h^{\prime}\left(x_{1}\right)=1, h \vee h^{\prime}\left(x_{i}\right)=0.6, i=2, \ldots, n \Rightarrow I\left(h \vee h^{\prime}\right)=0.84 .
\end{aligned}
$$

It is evident that $h \approx h^{\prime}$, but

$$
I\left(h \vee h^{\prime}\right)=0.84 \neq 0.6=I(h) \vee I\left(h^{\prime}\right) .
$$

Therefore the condition $\left(a^{\vee}\right)$ does not follow from (b), (c) and $\left(d^{\wedge}\right)$.

Example 3.5. Let us consider the functional

$$
I(h)= \begin{cases}\min _{1 \leqslant i \leqslant n} h\left(x_{i}\right) & \text { if } \max _{1 \leqslant i \leqslant n} h\left(x_{i}\right) \neq h\left(x_{n}\right), \\ \max \left(\min _{1 \leqslant i \leqslant n} h\left(x_{i}\right), \min \left(0.5, h\left(x_{n}\right)\right)\right) & \text { if } \max _{1 \leqslant i \leqslant n} h\left(x_{i}\right)=h\left(x_{n}\right) .\end{cases}
$$


It is easy to check that $I(\cdot)$ fulfills the conditions $\left(a^{\vee}\right),(c)$ and $\left(d^{\wedge}\right)$, but it does not fulfill (b); For example, if

$$
\begin{aligned}
& h\left(x_{i}\right)=0, i=1, \ldots, n-1, h\left(x_{n}\right)=0.6 \Rightarrow I(h)=0.5, \\
& h^{\prime}\left(x_{i}\right)=0, i=1, \ldots, n-2, h^{\prime}\left(x_{n-1}\right)=1, h^{\prime}\left(x_{n}\right)=0.8 \Rightarrow I\left(h^{\prime}\right)=0,
\end{aligned}
$$

then $h \leqslant h^{\prime}$ but $I(h)=0.5>0=I\left(h^{\prime}\right)$. So, the condition (b) cannot be deduced from $\left(a^{\vee}\right)$, (c) and $\left(d^{\wedge}\right)$.

Example 3.6. The functional

$$
I(h)=\min \left(0.5, \min _{1 \leqslant i \leqslant n} h\left(x_{i}\right)\right)
$$

obviously satisfies the conditons $\left(a^{\vee}\right),(b)$ and $\left(d^{\wedge}\right)$ but does not satisfy (c).

Example 3.7. Let $I(\cdot)$ be the functional

$$
I(h)=\max \left(\min _{1 \leqslant i \leqslant n} h\left(x_{i}\right), 0.5 \max _{1 \leqslant i \leqslant n} h\left(x_{i}\right)\right) .
$$

$I(\cdot)$ satisfies the conditions $\left(a^{\vee}\right)$, (b) and $(c)$, but it does not satisfy $\left(d^{\wedge}\right)$. For example, if

$$
\begin{aligned}
& a=0.6 \text { and } h\left(x_{1}\right)=0.2, h\left(x_{i}\right)=1, i=2, \ldots, n \Rightarrow I(h)=0.5, \\
& (a \wedge h)\left(x_{1}\right)=0.2,(a \wedge h)\left(x_{i}\right)=0.6, i=2, \ldots, n \Rightarrow I(a \wedge h)=0.3,
\end{aligned}
$$

and $a \wedge I(h)=0.5 \neq 0.3=I(a \wedge h)$.

Unlike Choquet's integral, the characterization theorem for Sugeno's integral cannot be refined by removing any property. This difference between both fuzzy integrals will be explained in the next section.

\section{Other fuzzy integrals}

As we have seen in Section 2, Choquet and Sugeno's integrals fit the following four basic properties model:

Functionals $I(\cdot)$ defined on functions $h: X \rightarrow Y$, where $Y=\mathbb{R}_{0}^{+}$or $Y=[0,1]$, satisfying

( $\left.\mathrm{a}^{\perp}\right)=h^{\prime} \Rightarrow I\left(h \perp h^{\prime}\right)=I(h) \perp I\left(h^{\prime}\right)$ (ordered $\perp$-additivity),

(b) $h \leqslant h^{\prime} \Rightarrow I(h) \leqslant I\left(h^{\prime}\right)$,

(c) $I\left(I_{X}\right)=1$,

$\left(\mathrm{d}^{*}\right) I(a * h)=a * I(h) \forall a \in Y-\{0\}$,

where $\perp$ and $*$ are binary operations on $Y$.

Concretely,

$$
\begin{aligned}
& Y=\mathbb{R}_{0}^{+}, \perp=+ \text { and } *=\cdot \Rightarrow I(\cdot) \equiv \text { Choquet's integral. } \\
& Y=[0,1], \perp=\vee \text { and } *=\wedge \Rightarrow I(\cdot) \equiv \text { Sugeno's integral. }
\end{aligned}
$$


Now, this raises the possibility of defining other fuzzy integrals of the same class but using different binary operations with similar properties to sum, product, maximum and minimum.

Note that, given $\perp$, if we use the following operation [14]:

$$
\forall a, b \in Y \quad a \Theta b=\inf \{c \in Y \mid c \perp b \geqslant a\},
$$

then, under some conditions, we can express any function $h$ as

$$
h(\cdot)=\stackrel{n}{\perp}\left(\left(h\left(x_{i}\right) \Theta h\left(x_{i-1}\right)\right) * I_{A_{i}}(\cdot)\right)
$$

where $h\left(x_{1}\right) \leqslant h\left(x_{2}\right) \leqslant \cdots \leqslant h\left(x_{n}\right), \quad A_{i}=\left\{x_{i}, x_{i+1}, \ldots, x_{n}\right\}, i=1, \ldots, n$, and $h\left(x_{0}\right)=0$.

For example, for $\perp=+$ and $*=\cdot$,

$$
a \ominus b= \begin{cases}a-b & \text { if } a>b, \\ 0 & \text { if } a \leqslant b,\end{cases}
$$

and

$$
h(\cdot)=\sum_{i=1}^{n}\left(\left(h\left(x_{i}\right) \ominus h\left(x_{i-1}\right)\right) I_{A_{i}}(\cdot)\right)=\sum_{i=1}^{n}\left(\left(h\left(x_{i}\right)-h\left(x_{i-1}\right)\right) I_{A_{i}}(\cdot)\right) .
$$

For $\perp=\vee$ and $*=\wedge$,

$$
a \ominus b= \begin{cases}a & \text { if } a>b, \\ 0 & \text { if } a \leqslant b,\end{cases}
$$

and

$$
h(\cdot)=\bigvee_{i=1}^{n}\left(\left(h\left(x_{i}\right) \Theta h\left(x_{i-1}\right)\right) \wedge I_{A_{i}}(\cdot)\right)=\bigvee_{i=1}^{n}\left(h\left(x_{i}\right) \wedge I_{A_{i}}(\cdot)\right)
$$

It is easy to check that the functions

$$
f_{i}(\cdot)=\left(h\left(x_{i}\right) \ominus h\left(x_{i-1}\right)\right) * I_{A_{i}}(\cdot)
$$

are equiordered between each other, $f_{i} \simeq f_{j}, i, j=1, \ldots, n$. Therefore any functional $I(\cdot)$ fulfilling the properties $\left(\mathrm{a}^{\perp}\right)(\mathrm{b}),(\mathrm{c})$ and $\left(\mathrm{d}^{*}\right)$ can be written as

$$
I(h)=\stackrel{\perp}{\perp}_{i=1}^{n}\left(\left(h\left(x_{i}\right) \ominus h\left(x_{i-1}\right)\right) * g\left(A_{i}\right)\right)
$$

where $g$ is a fuzzy measure defined as $g(A)=I\left(I_{A}\right) \forall A \subseteq X$.

Expression (6) is similar to (1) and (2).

Note also that results of the operation * are only relevant in the calculation of $I(\cdot)$ for those pairs in $Y \times D$, where $D=\left\{c \in Y \mid c=I\left(I_{A}\right)\right.$ for some $\left.A \subseteq X\right\}$.

To make the previous development, in order to assure the decomposition (5), $\perp$ and $*$ must satisfy at least the following properties:

1. $\perp$ and $*$ are monotonic.

2. $\perp$ is continuous and associative.

3. $a * 1=a$ and $a * 0=0 \forall a \in Y$.

4. $a \perp 0=a \forall a \in Y$. 
Nevertheless, these properties are not sufficient to assure the functional $I(\cdot)$ to be well-defined, as we will see later.

First, we will try to obtain new integrals by only replacing (in Choquet's and Sugeno's integrals respectively) the product and the minimum by an operation $*$, and preserving the sum and the maximum.

Proposition 4.1. If a functional $I: \mathscr{F}_{\infty} \rightarrow \mathbb{R}_{0}^{+}$satisfies the conditions $\left(\mathrm{a}^{+}\right),(\mathrm{b}),(\mathrm{c})$ and $\left(\mathrm{d}^{*}\right)$ then $*$ coincides with the product, at least for every pair in $Y \times D$.

Proof. Let $a \in \mathbb{R}_{0}^{+}, A \subseteq X$ and the function

$$
h\left(x_{i}\right)= \begin{cases}a & \text { if } x_{i} \in A, \\ 0 & \text { if } x_{i} \notin A .\end{cases}
$$

The function $h$ may be written as $h(\cdot)=a * I_{A}(\cdot)$, and therefore, using $\left(\mathrm{d}^{*}\right)$,

$$
I(h)=I\left(a * I_{A}\right)=a * I\left(I_{A}\right) .
$$

On the other hand, it is obvious that $h(\cdot)=a I_{A}(\cdot)$. As $I(\cdot)$ satisfies $\left(\mathrm{a}^{+}\right),(\mathrm{b})$ and (c), and taking into account Proposition 3.1, we can assure that $I(h)=I\left(a I_{A}\right)=$ $a I\left(I_{A}\right)$. Then

$$
a * I\left(I_{A}\right)=a I\left(I_{A}\right)
$$

and $*$ coincides with $\cdot$ on $Y \times D$.

As for every pair not included among those, results of the operation $*$ are not important at all for the calculation of $I(\cdot)$, then, in practice the product is the only binary operation compatible with $\left(\mathrm{a}^{+}\right),(\mathrm{b})$ and (c). Therefore, if we keep the sum, Choquet's integral cannot be generalized using operations different from the product.

The situation is very different for Sugeno's integral. This is a particular case of a type of integrals studied by Weber $[14,15]$ and Suarez [10], which we will call t-normed integrals:

$$
T_{g}(h, *)=\max _{0 \leqslant \alpha \leqslant 1}\left\{\alpha * g\left(H_{\alpha}\right)\right\} .
$$

The following theorem gives a characterization for this kind of integral:

Theorem 4.1. A functional $I: \mathscr{F}_{1} \rightarrow[0,1]$ satisfies the conditions $\left(\mathrm{a}^{\vee}\right),(\mathrm{b}),(\mathrm{c})$ and

$\left(\mathrm{d}^{*}\right) I(a * h)=a * I(h) \forall a \in(0,1]$, where $*$ is a t-norm [13],

if and only if a unique fuzzy measure $g$ exists such that $I(\cdot)$ is the t-normed integral with respect to $g$ for the $\mathrm{t}$-norm $*, T_{g}(\cdot, *)$.

Proof. Necessity: It can be proved (in a similar way as in [6]) that $g(A)=I\left(I_{A}\right)$ $\forall A \subseteq X$ is a fuzzy measure.

Consider a function $h: X \rightarrow[0,1]$ and let us suppose that it satisfies $h\left(x_{1}\right) \leqslant$ $h\left(x_{2}\right) \leqslant \cdots \leqslant h\left(x_{n}\right)$, which is not a restriction. Then $h$ can be expressed as

$$
h(\cdot)=\bigvee_{i=1}^{n}\left(h\left(x_{i}\right) * I_{A_{i}}(\cdot)\right)
$$


As functions $h\left(x_{i}\right) * I_{A_{i}}(\cdot)$ are equiordered between each other, using $\left(\mathrm{a}^{\vee}\right)$ and $\left(d^{*}\right)$ we obtain

$$
\begin{aligned}
I(h) & =I\left(\bigvee_{i=1}^{n}\left(h\left(x_{i}\right) * I_{A_{i}}\right)\right)=\bigvee_{i=1}^{n} I\left(h\left(x_{i}\right) * I_{A_{i}}\right) \\
& =\bigvee_{i=1}^{n}\left(h\left(x_{i}\right) * I\left(I_{A_{i}}\right)\right)=\bigvee_{i=1}^{n}\left(h\left(x_{i}\right) * g\left(A_{i}\right)\right),
\end{aligned}
$$

which is the expanded expression of $T_{g}(h, *)$.

Sufficiency: The conditions (b), (c) and $\left(\mathrm{d}^{*}\right)$ are well-known properties of t-normed integrals.

To prove (a $\mathrm{a}^{\vee}$ ), let $h$ and $h^{\prime}$ be two equiordered functions. If we again suppose that $h\left(x_{1}\right) \leqslant h\left(x_{2}\right) \leqslant \cdots \leqslant h\left(x_{n}\right)$, then $h^{\prime}\left(x_{1}\right) \leqslant \cdots \leqslant h^{\prime}\left(x_{n}\right)$ and $\left(h \vee h^{\prime}\right)\left(x_{1}\right) \leqslant$ $\cdots \leqslant\left(h \vee h^{\prime}\right)\left(x_{n}\right)$.

Using distributivity of any t-norm over the maximum operator,

$$
\begin{aligned}
T_{g}\left(h \vee h^{\prime}, *\right) & =\bigvee_{i=1}^{n}\left(\left(h\left(x_{i}\right) \vee h^{\prime}\left(x_{i}\right)\right) * g\left(A_{i}\right)\right) \\
& =\bigvee_{i=1}^{n}\left(\left(h\left(x_{i}\right) * g\left(A_{i}\right)\right) \vee\left(h^{\prime}\left(x_{i}\right) * g\left(A_{i}\right)\right)\right) \\
& =\bigvee_{i=1}^{n}\left(h\left(x_{i}\right) * g\left(A_{i}\right)\right) \vee \bigvee_{i=1}^{n}\left(h^{\prime}\left(x_{i}\right) * g\left(A_{i}\right)\right)=T_{g}(h, *) \vee T_{g}\left(h^{\prime}, *\right),
\end{aligned}
$$

and condition $\left(\mathrm{a}^{\vee}\right)$ holds.

So, Sugeno's integral can be generalized by replacing the minimum by any t-norm $*$, thus obtaining the t-normed integrals.

This difference between the possibilities of generalization of Choquet's and Sugeno's integral may be explained by means of the following result:

Proposition 4.2. If a functional $I(\cdot)$ satisfying the conditions $\left(\mathrm{a}^{\perp}\right),(\mathrm{b})$, (c) and $\left(\mathrm{d}^{*}\right)$ exists, then $(a \perp b) * c=(a * c) \perp(b * c) \forall a, b \in Y, \forall c \in D$, that is, * is distributive over $\perp$, at least for the values important for the calculation of $I(\cdot)$.

Proof. Let $a, b \in Y, A \subseteq X$ and two functions

$$
h\left(x_{i}\right)=\left\{\begin{array}{ll}
a & \text { if } x_{i} \in A, \\
0 & \text { otherwise, }
\end{array} \quad h^{\prime}\left(x_{i}\right)= \begin{cases}b & \text { if } x_{i} \in A, \\
0 & \text { otherwise }\end{cases}\right.
$$

It is obvious that $h \simeq h^{\prime}$. The function $h \perp h^{\prime}$ is

$$
\left(h \perp h^{\prime}\right)\left(x_{i}\right)= \begin{cases}a \perp b & \text { if } x_{i} \in A, \\ 0 & \text { otherwise. }\end{cases}
$$
$\left(d^{*}\right)$,

As $h(\cdot)=a * I_{A}(\cdot), h^{\prime}(\cdot)=b * I_{A}(\cdot)$ and $\left(h \perp h^{\prime}\right)(\cdot)=(a \perp b) * I_{A}(\cdot)$ then, using

$$
I(h)=a * I\left(I_{A}\right), \quad I\left(h^{\prime}\right)=b * I\left(I_{A}\right), \quad I\left(h \perp h^{\prime}\right)=(a \perp b) * I\left(I_{A}\right)
$$


But because of $\left(a^{\perp}\right)$,

$$
\left(a * I\left(I_{A}\right)\right) \perp\left(b * I\left(I_{A}\right)\right)=(a \perp b) * I\left(I_{A}\right),
$$

and $*$ is distributive over $\perp$ for the aforesaid values.

So, as other authors also pointed out $[9,12,14]$, the crucial property is a distribution law. Now it is clear why Choquet's integral cannot be generalized (replacing $\cdot$ ) whereas it is possible to do it (replacing $\wedge$ ) with Sugeno's integral:

In Choquet's integral case, $\perp=+$ and the only operation distributive over + among those considered is the product.

In Sugeno's integral case, $\perp=\vee$ and any t-norm is distributive over $\vee$.

If sum or maximum operations are fixed, the possibilities of extension become clear. Can we replace these operations to obtain a larger class of functionals? The answer depends on the properties required for $\perp$ and $*$.

If $Y=\mathbb{R}_{0}^{+}$we propose to use instead $\perp$ a class of binary operations more restrictive than the class of pseudo-additions [12]:

Consider a continuous and strictly increasing function $k: \mathbb{R}_{0}^{+} \rightarrow \mathbb{R}_{0}^{+}$such that $k(0)=0$ and $\lim _{x \rightarrow \infty} k(x)=+\infty$. Then we define the $k$-sum $\perp$ as

$$
a \perp b=k^{-1}(k(a)+k(b)) \quad \forall a, b \in \mathbb{R}_{0}^{+} .
$$

It is easy to prove the following properties of $\perp$ :

$\left(1^{\perp}\right) \perp$ is associative and commutative.

$\left(2^{\perp}\right) \perp$ is continuous and strictly increasing.

(3 $\left.3^{\perp}\right) \quad a \perp 0=a \forall a \in \mathbb{R}_{0}^{+}$.

$\left(4^{\perp}\right) k(a \perp b)=k(a)+k(b) \forall a, b \in \mathbb{R}_{0}^{+}$.

$$
a \ominus b= \begin{cases}k^{-1}(k(a)-k(b)) & \text { if } a>b, \\ 0 & \text { if } a \leqslant b .\end{cases}
$$

Therefore $\perp$ is an operation similar to the sum.

Because of Proposition 4.2, if we want to build a fuzzy integral using $\perp$, in accordance with our model, we need another operation $*$ distributive over $\perp$. We can obtain operations $*$ of this kind in the following way:

Consider a continuous and non-decreasing function $s: \mathbb{R}_{0}^{+} \rightarrow \mathbb{R}_{0}^{+}$such that $s(0)=0$, and $s(1)=1$. We define the $k-s$-product $*$ as

$$
a * b=k^{-1}(k(a) s(b)) \quad \forall a, b \in \mathbb{R}_{0}^{+} .
$$

It is also easy to check the following properties of $*$ :

$\left(1^{*}\right) *$ is non-decreasing and continuous.

(2*) $a * 1=a \forall a \in \mathbb{R}_{0}^{+}$.

(3*) $a * 0=0 \forall a \in \mathbb{R}_{0}^{+}$.

$\left(4^{*}\right)(a \perp b) * c=(a * c) \perp(b * c) \forall a, b, c \in \mathbb{R}_{0}^{+}$.

Using these operations, and given any fuzzy measure $g$, the functional

$$
T_{g}(h,(k, s))=\stackrel{n}{\perp}\left(\left(h\left(x_{i}\right) \ominus h\left(x_{i-1}\right)\right) * g\left(A_{i}\right)\right)
$$

where $h\left(x_{1}\right) \leqslant h\left(x_{2}\right) \leqslant \cdots \leqslant h\left(x_{n}\right), \quad A_{i}=\left\{x_{i}, x_{i+1}, \ldots, x_{n}\right\}, i=1, \ldots, n$, and $h\left(x_{0}\right)=0$, is well-defined, and we will call it a $k-s$-fuzzy integral. 
The following proposition shows the close relation existing between $k-s$-fuzzy integrals and Choquet's integral:

Proposition 4.3. The $k$-s-fuzzy integral of a function $h \in \mathscr{F}_{\infty}$ with respect to a fuzzy measure $g$ coincides with the inverse image using $k$ of Choquet's integral of the function $k \circ h$ with respect to the fuzzy measure $s \circ g(s \circ g(A)=s(g(A)))$ :

$$
T_{g}(h,(k, s))=k^{-1}\left(E_{s \circ g}(k \circ h)\right) .
$$

Proof. $s \circ g$ is a fuzzy measure because $s(0)=0, s(1)=1$ and $s$ is non-decreasing. Now

$$
\begin{aligned}
T_{g}(h,(k, s)) & =\underset{i=1}{\perp}\left(\left(h\left(x_{i}\right) \ominus h\left(x_{i-1}\right)\right) * g\left(A_{i}\right)\right) \\
& =\stackrel{i=1}{n}_{i=1}^{n}\left(k^{-1}\left(k\left(h\left(x_{i}\right) \ominus h\left(x_{i-1}\right)\right) s\left(g\left(A_{i}\right)\right)\right)\right) \\
& =\stackrel{\perp=1}{n}_{i=1}^{n}\left(k^{-1}\left(\left(k\left(h\left(x_{i}\right)\right)-k\left(h\left(x_{i-1}\right)\right)\right) s\left(g\left(A_{i}\right)\right)\right)\right) \\
& =k^{-1}\left(\sum_{i=1}^{n}\left(\left(k\left(h\left(x_{i}\right)\right)-k\left(h\left(x_{i-1}\right)\right)\right) s\left(g\left(A_{i}\right)\right)\right)\right) \\
& =k^{-1}\left(E_{s \mathrm{og}}(k \circ h)\right) .
\end{aligned}
$$

So, $k-s$-fuzzy integrals may be considered as modifications of Choquet's integral.

Nevertheless, $k-s$-fuzzy integrals do not satisfy the property ( $\mathrm{d}^{*}$ ) (because of non-associativity of $*$ and compatibility problems between $*$ and $\Theta$ ), although they satisfy the properties $\left(\mathrm{a}^{\perp}\right)$, (b) and (c). To obtain a characterization theorem similar to Theorems 2.12 .2 and 4.1 , it is necessary to consider only $k-k$-fuzzy integrals (in order to avoid the problems before).

Theorem 4.2. A functional $I: \mathscr{F}_{\infty} \rightarrow \mathbb{R}_{0}^{+}$satisfies the conditions $\left(\mathrm{a}^{\perp}\right),(\mathrm{b}),(\mathrm{c})$ and $\left(\mathrm{d}^{*}\right)$, where $\perp$ is a $k$-sum and $* a k-k$-product, if and only if a unique fuzzy measure $g$ exists such that $I(\cdot)$ is the $k-k$-fuzzy integral with respect to $g$, $T_{g}(.,(k, k))$.

Proof. Necessity: If we define $g(A)=I\left(I_{A}\right) \forall A \subseteq X$, from conditions (c) and (b), we have that $g(X)=1$ and the monotonicity of $g$. To assure that $g$ is a fuzzy measure it suffices to prove that $g(\emptyset)=0$ :

As $I_{\emptyset}(\cdot) \simeq I_{\emptyset}(\cdot)$, using $\left(\mathrm{a}^{\perp}\right)$,

Then

$$
I\left(I_{\emptyset}\right)=I\left(I_{\emptyset} \perp I_{\emptyset}\right)=I\left(I_{\emptyset}\right) \perp I\left(I_{\emptyset}\right) .
$$

$$
k\left(I\left(I_{\emptyset}\right)\right)=k\left(I\left(I_{\emptyset}\right) \perp I\left(I_{\emptyset}\right)\right)=k\left(I\left(I_{\emptyset}\right)\right)+k\left(I\left(I_{\emptyset}\right)\right) .
$$

Therefore $k\left(I\left(I_{\emptyset}\right)\right)=0 \Rightarrow g(\emptyset)=I\left(I_{\emptyset}\right)=0$, and $g$ is a fuzzy measure. 
Let us consider a function $h$ such that

$$
h\left(x_{1}\right) \leqslant h\left(x_{2}\right) \leqslant \cdots \leqslant h\left(x_{n}\right) .
$$

Then we can write $h$ as

$$
h(\cdot)=\stackrel{\perp}{\perp}=1_{\perp}\left(\left(h\left(x_{i}\right) \ominus h\left(x_{i-1}\right)\right) * I_{A_{i}}(\cdot)\right),
$$

and using $\left(\mathrm{a}^{\perp}\right)$ and $\left(\mathrm{d}^{*}\right)$,

$$
I(h)=\stackrel{\perp}{\perp}=1_{\perp}\left(\left(h\left(x_{i}\right) \ominus h\left(x_{i-1}\right)\right) * g\left(A_{i}\right)\right)=T_{g}(h,(k, k)) .
$$

Sufficiency: We will use Proposition 4.3 and properties of Choquet's integral to prove the conditions $\left(\mathrm{a}^{\perp}\right),(\mathrm{b}),(\mathrm{c})$ and $\left(\mathrm{d}^{*}\right)$.

If $h \simeq h^{\prime}, k \circ h \simeq k \circ h^{\prime}$ because $k$ is increasing; then

$$
\begin{aligned}
T_{g}\left(h \perp h^{\prime},(k, k)\right) & =k^{-1}\left(E_{k \circ g}\left(k \circ\left(h \perp h^{\prime}\right)\right)\right)=k^{-1}\left(E_{k \circ g}\left(k \circ h+k \circ h^{\prime}\right)\right) \\
& =k^{-1}\left(E_{k \circ g}(k \circ h)+E_{k \circ g}\left(k \circ h^{\prime}\right)\right) \\
& =k^{-1}\left(k\left(T_{g}(h,(k, k))\right)+k\left(T_{g}\left(h^{\prime},(k, k)\right)\right)\right) \\
& =T_{g}(h,(k, k)) \perp T_{g}\left(h^{\prime},(k, k)\right) .
\end{aligned}
$$

If $h \leqslant h^{\prime}, k \circ h \leqslant k \circ h^{\prime}$, then

Finally,

$$
\begin{aligned}
T_{g}(h,(k, k)) & =k^{-1}\left(E_{k \circ g}(k \circ h)\right) \leqslant k^{-1}\left(E_{k \circ g}\left(k \circ h^{\prime}\right)\right)=T_{g}\left(h^{\prime},(k, k)\right) . \\
T_{g}\left(I_{X},(k, k)\right) & =k^{-1}\left(E_{k \circ g}\left(k \circ I_{X}\right)\right)=k^{-1}\left(E_{k \circ g}\left(k(1) I_{X}\right)\right) \\
& =k^{-1}\left(k(1) E_{k \circ g}\left(I_{X}\right)\right)=k^{-1}(k(1))=1 .
\end{aligned}
$$

$$
\begin{aligned}
T_{g}(a * h,(k, k)) & =k^{-1}\left(E_{k \circ g}(k \circ(a * h))\right)=k^{-1}\left(E_{k \circ g}(k(a)(k \circ h))\right) \\
& =k^{-1}\left(k(a) E_{k \circ g}(k \circ h)\right)=k^{-1}\left(k(a) k\left(T_{g}(h,(k, k))\right)\right) \\
& =a * T_{g}(h,(k, k)) .
\end{aligned}
$$

Therefore $T_{g}(\cdot,(k, k))$ satisfies $\left(\mathrm{a}^{\perp}\right),(\mathrm{b}),(\mathrm{c})$ and $\left(\mathrm{d}^{*}\right)$.

Next, we give some examples of $k-s$-fuzzy integrals:

Example 4.1. Consider the functions $k(x)=\ln (1+x)$ and $s(x)=x$. Then

$$
\begin{aligned}
& k^{-1}(x)=\exp (x)-1, \\
& a \perp b=a+b+a b, \\
& a \ominus b=(a-b) /(1+b) \quad(\text { if } a \geqslant b), \\
& a * b=(1+a)^{b}-1 .
\end{aligned}
$$

The associated $k$-s-fuzzy integral is

$$
T_{g}(h,(k, s))=\prod_{i=1}^{n}\left(\left(1+h\left(x_{i}\right)\right)^{g\left(A_{i}\right)-g\left(A_{i+1}\right)}\right)-1 .
$$

Note that $\ln \left(1+T_{g}(h,(k, s))\right)=E_{g}(\ln (1+h))$. 
Example 4.2. Consider the function $k(x)=\log _{2}(1+x)=\ln (1+x) / \ln (2)$. Then

$$
\begin{aligned}
& k^{-1}(x)=2^{x}-1=\exp (x \ln (2))-1, \\
& a \perp b=a+b+a b, \\
& a \ominus b=(a-b) /(1+b) \quad(\text { if } a \geqslant b), \\
& a * b=\exp (\ln (1+a) \ln (1+b) / \ln (2))-1 .
\end{aligned}
$$

The associated $k-k$-fuzzy integral is

$$
T_{g}(h,(k, k))=\prod_{i=1}^{n}\left(\left(\frac{1+h\left(x_{i}\right)}{1+h\left(x_{i-1}\right)}\right)^{\log _{2}\left(1+g\left(A_{i}\right)\right)}\right)-1 .
$$

Note that $\log _{2}\left(1+T_{g}(h,(k, k))\right)=E_{\log _{2}(1+g(\cdot))}\left(\log _{2}(1+h)\right)$.

Example 4.3. Consider the function $k(x)=x^{p}, p>0$. Then

$$
\begin{aligned}
& K^{-1}(x)=(x)^{1 / p}, \\
& a \perp b=\left(a^{p}+b^{p}\right)^{1 / p}, \\
& a \ominus b=\left(a^{p}-b^{p}\right)^{1 / p} \quad(\text { if } a \geqslant b), \\
& a * b=a b .
\end{aligned}
$$

The associated $k-k$-fuzzy integral is

$$
T_{g}(h,(k, k))=\left(\sum_{i=1}^{n}\left(\left(h^{p}\left(x_{i}\right)-h^{p}\left(x_{i-1}\right)\right) g^{p}\left(A_{i}\right)\right)\right)^{1 / p}
$$

Note also that $\left(T_{g}(h,(k, k))\right)^{p}=E_{g^{p}}\left(h^{p}\right)$.

Next, we will show how replacing the condition $a^{\perp}$ in the Theroem 4.2 by a more restrictive condition we obtain integrals for $\perp$-decomposable measures [14]:

Proposition 4.4. If in Theorem 4.2 the condition $a^{\perp}$ is replaced by the more restrictive condition of $\perp$-additivity:

$$
I\left(h \perp h^{\prime}\right)=I(h) \perp I\left(h^{\prime}\right) \quad \forall h, h^{\prime} \in \mathscr{F}_{\infty},
$$

then and only then $I(\cdot)$ is a $k$-k-fuzzy integral with respect to a $\perp$-decomposable measure.

Proof. Necessity: As $\forall A, B \subseteq X$ with $A \cap B=\emptyset, I_{A \cup B}(\cdot)=I_{A}(\cdot) \perp I_{B}(\cdot)$, then

$$
g(A \cup B)=I\left(I_{A \cup B}\right)=I\left(I_{A} \perp I_{B}\right)=I\left(I_{A}\right) \perp I\left(I_{B}\right)=g(A) \perp g(B),
$$

and $g$ is a $\perp$-decomposable measure.

Sufficiency: If $g$ is a $\perp$-decomposable measure, taking into account the properties of $\perp$ and $*$, the expression

$$
I(h)=\stackrel{n}{\perp}\left(\left(h\left(x_{i}\right) \ominus h\left(x_{i-1}\right)\right) * g\left(A_{i}\right)\right)
$$


of $I(\cdot)$ can be rewritten in the following way:

So,

$$
I(h)=\stackrel{n}{\perp}\left(\left(h\left(x_{i}\right) * g\left(\left\{x_{i}\right\}\right)\right) .\right.
$$

$$
\begin{aligned}
& I\left(h \perp h^{\prime}\right)=\stackrel{\perp}{i=1}_{\perp}\left(\left(h\left(x_{i}\right) \perp h^{\prime}\left(x_{i}\right)\right) * g\left(\left\{x_{i}\right\}\right)\right) \\
& =\stackrel{i}{i=1}_{\perp}^{n}\left(\left(h\left(x_{i}\right) * g\left(\left\{x_{i}\right\}\right)\right) \perp\left(h^{\prime}\left(x_{i}\right) * g\left(\left\{x_{i}\right\}\right)\right)\right) \\
& =\underset{i=1}{\perp}\left(h\left(x_{i}\right) * g\left(\left\{x_{i}\right\}\right)\right) \perp \underset{i=1}{\perp}\left(h^{\prime}\left(x_{i}\right) * g\left(\left\{x_{i}\right\}\right)\right)=I(h) \perp I\left(h^{\prime}\right),
\end{aligned}
$$

and condition (9) holds.

Campos and Bolaños [6] proved that Choquet's and Sugeno's integrals are natural extensions to general fuzzy measures of the mathematical expectation for probability measures and Sugeno's integral for possibility measures respectively, as a consequence of weakening additivity and F-additivity by ordered additivity and ordered F-additivity. The same holds good for $\mathbf{k}-\mathbf{k}$-fuzzy integrals: they are the natural extension to fuzzy measures of integrals for $\perp$-decomposable measures, by replacing $\perp$-additivity by ordered $\perp$-additivity.

Finally, a similar study could be made for the case $Y=[0,1]$. However, note that if we want to obtain different fuzzy integrals, we cannot use t-conorms [13], instead of $\perp$, because of the necessity of a distributive law: the only t-conorm satisfying a distributive property with an operation $*$ is the maximum, and then we do not obtain new fuzzy integrals but $t$-normed integrals.

\section{Concluding remarks}

We have deeply studied how to build fuzzy integrals on general fuzzy measures. Our integration model is quite wide, and it includes most of the known fuzzy integrals. It takes as basis the generalization of a kind of additivity property by means of the equiordering relation.

Anyway, because of Proposition 4.3, it seems there are two really essential techniques of integration (as has been also pointed out by Murofushi and Sugeno [9]): a technique of additive average represented by Choquet's integral, and a technique of ordering, which is reflected by the t-normed integrals.

In order to use fuzzy integrals, it would be necessary to choose carefully the most appropriate type of integral for each problem, even making correlations between experimental and theoretical data.

Finally, another interesting matter would be to try to extend results of this paper to infinite referentials. 


\section{References}

[1] M. Berres, On a multiplication and a theory of integration for belief and plausibility functions, $J$. Math. Anal. Appl. 121 (1987) 487-505.

[2] M.J. Bolaños, M.T. Lamata and S. Moral, La esperanza monótona: una generalizacion de la esperanza probabilistica, In: Actas de la XV Reunion Nacional de la SEEIO, Asturias (1985) $57-64$.

[3] M.J. Bolaños, M.T. Lamata and S. Moral, Decision making problems in a general environment, Fuzzy Sets and Systems 25 (1988) 135-144.

[4] M.J. Bolaños, L.M. de Campos and A. Gonzalez, Convergence properties of the monotone expectation and its application to the extension of fuzzy measures, Fuzzy Sets and Systems 33 (1989) 201-212.

[5] L.M. de Campos and M.J. Bolaños, Representation of fuzzy measures through probabilities, Fuzzy Sets and Systems 31 (1989) 23-36.

[6] L.M. de Campos and M.J. Bolaños, Characterization and comparative study of two fuzzy integrals, J. Math. Anal. Appl., submitted.

[7] G. Choquet, Theory of capacities, Ann. Inst. Fourier 5 (1953) 131-295.

[8] R. Kruse, Fuzzy integrals and conditional fuzzy measures, Fuzzy Sets and Systems 10 (1983) 309-313.

[9] T. Murofushi and M. Sugeno, Fuzzy t-conorm integral with respect to fuzzy measures: generalization of Sugeno integral and Choquet integral, Fuzzy Sets and Systems, to appear.

[10] F. Suarez, Familias de integrales difusas y medidas de entropia relacionadas, Thesis, Universidad de Oviedo, Oviedo (1983).

[11] M. Sugeno, Theory of fuzzy integrals and its applications, Thesis, Tokyo Institute of Technology (1974).

[12] M. Sugeno and T. Murofushi, Pseudo-additive measures and integrals, J. Math. Anal. Appl. 122 (1987) 197-222.

[13] B. Schweizer and A. Sklar, Probabilistic Metric Spaces (North-Holland, New York, 1983).

[14] S. Weber, $\perp$-Decomposable measures and integrals for archimedean $t$-conorms $\perp, J$. Math. Anal. Appl. 101 (1984) 114-138.

[15] S. Weber, Two integrals and some modified versions - Critical remarks, Fuzzy Sets and Systems 20 (1986) 97-105. 